\title{
Silent Cerebral Infarction in Hemodialysis Patients
}

\author{
Tatsuya Nakatani Toshihide Naganuma J unji Uchida Chikayoshi Masuda \\ Seiji Wada Takeshi Sugimura Kazunobu Sugimura
}

Department of Urology, Osaka City University Medical School, Osaka, J apan

\section{Key Words}

Magnetic resonance imaging $\cdot$ Silent cerebral infarction .

Cerebrovascular disease $\cdot$ Hemodialysis

\begin{abstract}
Background: Cerebrovascular diseases are very common in hemodialysis (HD) patients. Silent cerebral infarction $(\mathrm{SCl})$ has not been investigated in $\mathrm{HD}$ patients although it may be a significant risk factor for cerebrovascular diseases. Hypothesis: Chronic renal failure may be an independent risk factor for $\mathrm{SCl}$ and cerebrovascular diseases. Methods: Cranial magnetic resonance imaging (MRI) was performed on $123 \mathrm{HD}$ patients without symptomatic cerebrovascular disease and on 52 control subjects. We investigated the prevalence of $\mathrm{SCl}$ and performed cross-sectional study using multiple logistic analysis to assess the relationship between $\mathrm{SCl}$ and the risk factors. Results: The prevalence of $\mathrm{SCl}$ was significantly higher in HD patients than in the healthy control group ( 60 patients $\left(48.8 \%\right.$ ) vs. 5 patients $(9.6 \%), \chi^{2}=22.4$, $\mathrm{p}<0.0001)$. Multiple logistic regression analysis with all subjects showed that independent risk factors of $\mathrm{SCl}$ were chronic renal failure, hypertension, smoking and age $\left(R^{2}=0.468, p<0.0001\right)$. In only the HD patient group, age and smoking were shown to be independent risk factors of $\mathrm{SCl}\left(\mathrm{R}^{2}=0.378, \mathrm{p}<0.0001\right)$ while HD duration and
\end{abstract}

\section{KARGER}

Fax +41613061234 E-Mail karger@karger.ch www. karger.com
(C) 2003 S. Karger AG, Base

0250-8095/03/0232-0086\$19.50/0

Accessible online at:

www. karger.com/ajn hypertension were not. Conclusions: The findings of the present study indicate that chronic renal failure maintained by hemodialysis increases the prevalence of $\mathrm{SCl}$ and that age and smoking habits are also significantly associated with $\mathrm{SCl}$ in $\mathrm{HD}$ patients.

Copyright @2003S. Karger AG, Basel

\section{Introduction}

Cerebrovascular disease is one of the major causes of death in hemodialysis (HD) patients [1-3]. It has been reported that HD patients have a much higher incidence of stroke than normal populations [1-4]. Strokes in HD patients are characterized by the high rate of intracerebral hemorrhage, and hypertension is also a significant risk factor for stroke in HD patients [1-4].

Silent cerebral infarction (SCI) is thought to be an underlying or concomitant condition of clinical subcortical brain infarction or brain hemorrhage [5]. In most cases SCI is found as a lacunar infarction, the most common form of subcortical infarction, defined by Fisher as small, deep cerebral infarction caused by occlusion of small penetrating cerebral arteries [6]. The lacunar infarction results from arteriolo-sclerotic change of cerebral arteries, which is related to aging and is accelerated by hypertension [7]. 
In spite of the clinical significance of asymptomatic SCI as a risk factor of stroke, little is known about SCI in HD patients. The influence of uremia and maintenance hemodialysis on SCI has not been elucidated yet. In the present study, we investigated the prevalence of SCI in HD patients and control subjects by magnetic resonance imaging (MRI) and analyzed the risk factors.

\section{Subjects and Methods}

\section{Study Design}

We performed a cross-sectional study using multiple logistic analysis to investigate the association between risk factors and SCI.

\section{Patients}

All subjects had no past history or symptoms of stroke, transient ischemic attacks or dementia. Table 1 shows patient characteristics in 123 HD patients and 52 normal controls. All HD patients received regular dialysis using the high-flux cellulose-triacetate or polysulfone hollow-fiber dialyzer three times per week in sessions lasting 3-4.5 h. The dialysate was buffered bicarbonate and contained $140 \mathrm{mmol} / \mathrm{l}$ sodium. The dialysate flow rate was $500 \mathrm{ml} / \mathrm{min}$, and blood flow ranged from 120 to $200 \mathrm{ml} / \mathrm{min}$. The dry weight was individually determined for each patient on the basis of post-HD cardiothoracic ratio, atrial natriuretic peptide and clinical observations such as presence of muscle cramps, general fatigue, thirst, or hypotension during the HD session, and all patients were maintained at their set dry weight. The control group consisted of 52 healthy subjects who visited our medical checkup clinic voluntarily for a multiphased health screening of the brain, physical examination, electrocardiography, chest X-ray, liver, renal function, glucose, lipids blood tests, MRI and magnetic resonance angiography scan of brain. There was no patient with renal dysfunction in the control group. Informed consent was obtained from all patients and control volunteers for participation in the study.

Hematocrit (\%) was significantly lower and prevalence of hypertension was significantly higher in HD patients than in the control group. On the other hand, the prevalence of dyslipidemia in HD patients was significantly lower than in the control group, which may be the result of malnutrition in HD patients.

\section{Risk Factor}

To evaluate risk factors, we investigated the presence or absence of hypertension, diabetes mellitus, dyslipidemia, ischemic heart disease, atrial fibrillation and smoking habits. Hypertension was defined by the administration of antihypertensive agents, a systolic blood pressure greater than $160 \mathrm{~mm} \mathrm{Hg}$ or a diastolic blood pressure greater than $95 \mathrm{~mm} \mathrm{Hg}$. Blood pressure was determined at the end point of HD session with a standard mercury sphygmomanometer and cuffs adapted to arm circumference. The systolic blood pressure was taken as the point of appearance of Korotkoff sounds, and the diastolic blood pressure as the point of disappearance of the sounds.

Dyslipidemia was defined as present if the subject had a total cholesterol $(\mathrm{TC})>220 \mathrm{mg} / \mathrm{dl}$, a triglyceride $(\mathrm{TG})>150 \mathrm{mg} / \mathrm{dl}$ and high-density lipoprotein (HDL), cholesterol $<40 \mathrm{mg} / \mathrm{dl}$, or had received medical treatment for hyperlipidemia. In HD patients blood samples were taken from the arterial line before HD sessions.
Table 1. Patients characteristics

\begin{tabular}{lclc}
\hline & $\begin{array}{l}\text { HD } \\
(\mathrm{n}=123)\end{array}$ & $\begin{array}{l}\text { Control } \\
(\mathrm{n}=52)\end{array}$ & $\begin{array}{l}\mathrm{p} \\
\text { value }\end{array}$ \\
\hline Age, years & $55.6 \pm 12.3$ & $51.7 \pm 12.2$ & $\mathrm{NS}$ \\
Males/females & $80 / 43$ & $29 / 23$ & $\mathrm{NS}$ \\
Hematocrit, \% & $30.5 \pm 3.2$ & $41.8 \pm 4.7$ & $<0.0001$ \\
Hypertension & 104 & 10 & $<0.0001$ \\
Diabetes mellitus & 35 & 9 & $\mathrm{NS}$ \\
Dyslipidemia & 16 & 24 & $<0.0001$ \\
Ischemic heart disease & 19 & 2 & $\mathrm{NS}$ \\
Atrial fibrillation & 9 & 0 & $\mathrm{NS}$ \\
Smoking habit & 32 & 9 & $\mathrm{NS}$ \\
Dialysis duration, years & $5.9 \pm 5.4$ & & \\
\hline
\end{tabular}

In healthy volunteers, blood samples were taken after resting supine for $10 \mathrm{~min}$.

Ischemic heart disease (IHD): Seventeen patients had stable exertional angina which was diagnosed by exercise ECG or myocardial perfusion imaging. Two patients had undergone coronary artery bypass surgery and the other 2 patients had old myocardial infarctions. All 21 patients received conventional treatment including vasodilators or calcium blockers.

Atrial fibrillation (AF): Three patients had persistent AF and 6 patients had paroxysmal AF. AF was diagnosed by at least two consecutive electrocardiographs administered more than $24 \mathrm{~h}$ apart. In all cases the heart rate was controlled within normal range by digitalis, calcium blockers, antiarrhythmic agents or pacemaker.

Body mass index was calculated as weight in kilograms divided by the square of height in meters.

\section{Magnetic Resonance Imaging}

All participating patients had a brain MRI that used a superconducting magnet at a field strength of $1.5 \mathrm{~T}$ on proton density, $\mathrm{T}_{1^{-}}$, and $\mathrm{T}_{2}$-weighted images in axial planes at $10 \mathrm{~mm}$ thick slices. Infarction was defined as a focal area $>5 \mathrm{~mm}$ in diameter in both $\mathrm{T}_{1^{-}}$and $\mathrm{T}_{2^{-}}$ weighted images which was visible as low-signal intensity areas on $\mathrm{T}_{1}$-weighted image and as high signal intensity areas on $\mathrm{T}_{2}$-weighted image. The MR images were assessed independently by two neuroradiologists. Cases with inconsistencies were excluded from the study.

\section{Statistical Analysis}

All data are presented as the mean \pm SD. Differences between groups were examined with Student's t tests. Categorical variables were compared using $\chi^{2}$ analysis. Multiple logistic regression analysis was used to assess the combined influence of variables on SCI. Gender, hypertension, diabetes mellitus, dyslipidemia, IHD, AF, smoking habit and chronic renal failure were represented by dummy variables $(1=$ male, $0=$ female; $1=$ presence, $0=$ absence $)$ in logistic analysis. A value of $\mathrm{p}<0.05$ was considered statistically significant. These results were obtained on a Macintosh computer using the Stat View V Statistical System. 


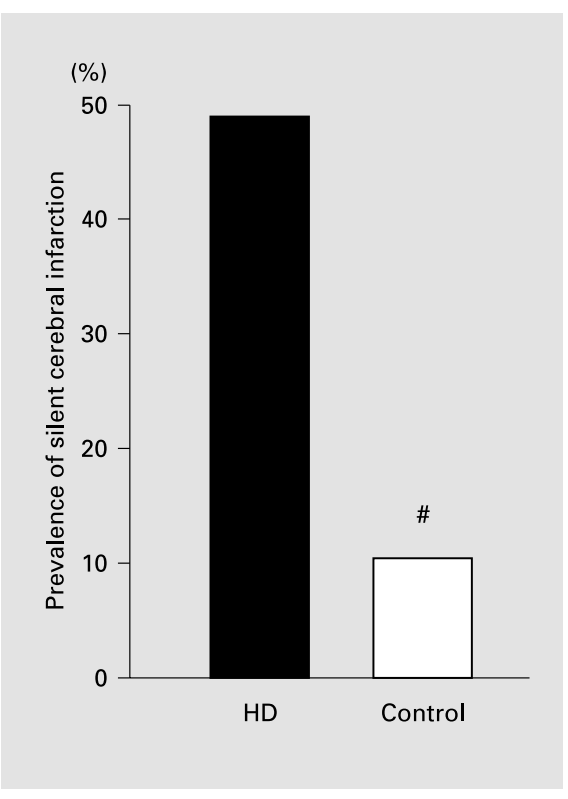

Fig. 1. Comparison of the prevalence of SCI between hemodialysis patients and healthy control group. ${ }^{\#} \mathrm{p}<0.0001$ compared to hemodialysis patients.

\section{Results}

Prevalence of SCI in HD Patients and Control Subjects

The prevalence of SCI was significantly higher in HD patients than in the healthy control group (60 patients $(48.8 \%)$ versus 5 patients $\left.(9.6 \%), \chi^{2}=22.4, \mathrm{p}<0.0001\right)$ (fig. 1). In $60 \mathrm{HD}$ patient with SCI, 57 cases had lacunar infarction and 3 cases had cortical infarction. In 5 cases with SCI in the control group, 4 cases had lacunar infarction and one had a cortical infarction.

\section{Multiple Logistic Regression Analysis of SCI (Model 1)}

In all subjects, multiple logistic regression analysis showed that independent risk factors of SCI were chronic renal failure, hypertension, smoking habit and age $\left(\mathrm{R}^{2}=\right.$ $0.468, \mathrm{p}<0.0001)($ table 2$)$

\section{Multiple Logistic Regression Analysis of SCI in HD Patients (Model 2)}

In HD patients only, multiple logistic regression analysis showed that independent risk factors of SCI were age and smoking habits $\left(\mathrm{R}^{2}=0.378, \mathrm{p}<0.0001\right)$ (table 3$)$. In contrast to model 1 , hypertension was not shown as an independent risk factor of SCI in HD patients.

Table 3. Risk factors affecting SCI in HD patients (model 2)

Table 2. Risk factors affecting SCI in all subjects (model 1)

\begin{tabular}{lllll}
\hline Variable & & \multirow{2}{*}{$\chi^{2}$} & $\mathrm{R}^{2}$ & $\mathrm{p}$ value \\
\cline { 1 - 2 } dependent & independent & & & \\
& & & & \\
& Age & $26.034^{* * *}$ & & \\
& Sex & 0.001 & & \\
& Hematocrit & 0.278 & & \\
& Hypertension & $9.160^{* *}$ & & \\
SCI & Diabetes mellitus & 0.219 & & \\
& Dyslipidemia & 3.204 & 0.468 & $<0.0001$ \\
& Ischemic heart disease & 1.383 & & \\
& Atrial fibrillation & 0.540 & & \\
& Smoking habit & $3.905^{*}$ & & \\
& Body mass index & 0.021 & & \\
& Chronic renal failure & $5.600^{*}$ & &
\end{tabular}

Significant predictors of silent cerebral infarction (SCI) were explored among the parameters including age, sex (female $=0$, male $=1)$, hematocrit, hypertension (absent $=0$, present $=1)$, diabetes mellitus (absent $=0$, present $=1)$, dyslipidemia $($ absent $=0$, present $=1)$, ischemic heart disease (absent $=0$, present $=1$ ), atrial fibrillation $($ absent $=0$, present $=1)$, smoking habit $($ absent $=0$, present $=1$ ), body mass index and chronic renal failure (absent $=0$, present $=1$ ). Final results were given in the table. $R^{2}$ is multiple coefficient of determination. ${ }^{* * *} \mathrm{p}<0.0001,{ }^{* *} \mathrm{p}<0.01,{ }^{*} \mathrm{p}<0.05$.

\begin{tabular}{lllll}
\hline Variable & & \multirow{2}{*}{$\chi^{2}$} & $\mathrm{R}^{2}$ & $\mathrm{p}$ value \\
\cline { 1 - 3 } dependent & independent & & & \\
& & & & \\
& Age & 0.009 & & \\
& Sex & 0.057 & & \\
& Hematocrit & 1.691 & & \\
& Hypertension & 0.590 & & \\
SCI & Diabetes mellitus & 1.841 & 0.378 & $<0.0001$ \\
& Dyslipidemia & 1.129 & & \\
& Ischemic heart disease & 0.283 & & \\
& Atrial fibrillation & $4.635^{*}$ & & \\
& Smoking habit & 0.097 & & \\
& Body mass index & 0.418 & &
\end{tabular}

Significant predictors of silent cerebral infarction (SCI) were explored among the parameters including age, sex (female $=0$, male $=1)$, hematocrit, hypertension (absent $=0$, present $=1$ ), diabetes mellitus (absent $=0$, present $=1)$, dyslipidemia $($ absent $=0$, present $=1)$, ischemic heart disease $($ absent $=0$, present $=1)$, atrial fibrillation (absent $=0$, present $=1)$, smoking habit $($ absent $=0$, present $=1$ ), body mass index and dialysis duration. Final results were given in the table. $\mathrm{R}^{2}$ is multiple coefficient of determination. $* * \mathrm{p}<0.0001, * \mathrm{p}<0.05$
Nakatani/Naganuma/Uchida/Masuda/

Wada/Sugimura/Sugimura 


\section{Discussion}

SCI is considered an important risk factor of stroke and there are several reports concerning SCI in the general population. From the results of MRI studies, Kobayashi et al. [5] reported that the incidence of SCI was 10.6\% in 993 neurologically normal adults without a history of cerebrovascular diseases. Howard et al. [8] also reported that the incidence of SCI was $11 \%$ using MRI studies. The Hisayama community-based study showed that the incidence of SCI was $12.9 \%$ [9]. These results are comparable with $9.6 \%$ of SCI prevalence in our control group.

In spite of the high incidence of stroke in HD patients, there has been no study concerning SCI in HD patients. This study is the first report to evaluate the incidence and risk factor of SCI in HD patients, showing the prevalence of SCI in HD patients to be 5 times greater than in the control group. The high incidence of hypertensive intracerebral hemorrhage in HD patients is well known [1-3]. Hypertensive intracerebral hemorrhage is thought to be based on arteriolosclerosis as well as the etiology of SCI. Kobayashi et al. [5] observed in their study that strokes occurring in subjects with SCI were due to clinical subcorticoid brain infarction or brain hemorrhage, suggesting that SCI may be an important marker for predicting those particular cerebrovascular diseases. Our findings regarding such a high incidence of SCI may be associated with the high incidence of intracerebral hemorrhage in HD patients. Further study will elucidate the role of SCI by correlating the rate of symptomatic ischemic or hemorrhagic stroke with SCI in HD patients who were otherwise neurologically normal at the beginning of the study.

Although the present study showed that chronic renal failure is significantly associated with SCI, the prevalence of SCI is not related to the duration of hemodialysis. Because SCI was measured as a logistic variable, not a timed variable, analysis of the factors related to time may not be reliable with this study design. However, these findings are consistent with the results of previous studies that atherosclerosis of aorta, carotid or femoral artery in HD patients are advanced, compared with healthy subjects [10-12] and that atherosclerosis may not be accelerated by hemodialysis itself $[10,13]$. Moreover, Jungers et al. [14] observed that the incidence of atherosclerotic cardiovascular complications is abnormally high in pre-dialysis chronic renal failure patients. Until SCI and the risk factors are examined in pre-dialysis patients, it remains unclear whether arteriolosclerosis leading to SCI is accelerated by end-stage renal failure without hemodialysis.
This study revealed that hypertension, age and smoking habits are significantly associated with SCI, which is consistent with previous studies in the general population. Kobayashi et al. [5] showed that hypertension, diabetes, alcohol habits $\geq 58 \mathrm{~g}$ per day, retinal artery sclerosis and age were significant and independent risk factors for subcortical silent brain infarction by multiple logistic regression analysis. Howard et al. [8] indicated hypertension, race, age and smoking habits as risk factors of SCI. On the other hand, our multiple logistic regression analysis in HD patients (model 2) showed only age and smoking as independent risk factors of SCI. The different results concerning hypertension between models 1 and 2 may be due to the difference in prevalence of hypertension in HD patients and in the control subjects. Whether hypertension is well controlled or not may also influence the results. As HD patients have many factors distinct from normal subjects that overlap and influence each other, it may be difficult to detect hypertension as an independent risk factor.

In conclusion, the findings of the present study indicate that chronic renal failure maintained by hemodialysis increases the prevalence of SCI and that age and smoking habits are also significantly associated with SCI in HD patients.

\section{Acknowledgements}

This work was supported by grants from the Osaka City University Medical Research Foundation. 


\section{References}

1 Iseki K, Kinjo K, Kimura Y, Osawa A, Fukiyama K, Nishime K, Uehara H, Tokuyama K, Toma S, Yoshihara K, Kowatari T, Terukina S, Kawazoe N: Evidence for high risk of cerebral hemorrhage in chronic dialysis patients. Kidney Int 1993;44:1086-1090.

2 Onoyama K, Ibayashi S, Nanishi F, Okuda S, Oh Y, Hirakata H, Nishimura Y, Fujishima M: Cerebral hemorrhage in patients on maintenance hemodialysis: CT analysis of 25 cases. Eur Neurol 1987;26:171-175.

3 Onoyama K, Kumagai H, Miishima T, Tsuruda H, Tomooka S, Motomura K, Fujishima M: Incidence of strokes and its prognosis in patients on maintenance hemodialysis. Jpn Heart J 1986;27:685-691.

4 Iseki K, Fukiyama K: Predictors of stroke in patients receiving chronic hemodialysis. Kidney Int 1996;50:1672-1675.

5 Kobayashi S, Okada K, Koide H, Bokura H, Yamaguchi S: Subcortical silent brain infarction as a risk factor for clinical stroke. Stroke 1997;28:1932-1939.
6 Fisher CM: Lacunar strokes and infarcts: A review. Neurology 1982;32:871-876.

7 Bruno A, Rosenberg GA: The spectrum of lacunar infarction in the elderly. Clin Geriatr Med 1991;7:443-453.

8 Howard G, Wagenknecht LE, Cai J, Cooper L, Kraut MA, Toole JF: Cigarette smoking and other risk factors for silent cerebral infarction in the general population. Stroke 1998;29:913917.

9 Shinkawa A, Ueda K, Kiyohara Y, Kato I, Sueishi K, Tsuneyoshi M, Fujishima M: Silent cerebral infarction in a community-based autopsy series in Japan: The Hisayama Study. Stroke 1995;26:380-385.

10 Kawagishi T, Nishizawa Y, Konishi T, Kawasaki K, Emoto M, Shoji T, Tabata T, Inoue T, Morii H: High-resolution B-mode ultrasonography in evaluation of atherosclerosis in uremia. Kidney Int 1995;48:820-826.
11 Shoji T, Kawagishi T, Emoto M, Maekawa K, Taniwaki H, Kanda H, Nishizawa Y: Additive impacts of diabetes and renal failure on carotid atherosclerosis. Atherosclerosis 2000;153:257258.

12 Shoji T, Nishizawa Y, Kawagishi T, Kawasaki $\mathrm{K}$, Taniwaki $\mathrm{H}$, Tabata $\mathrm{T}$, Inoue $\mathrm{T}$, Morii $\mathrm{H}$ : Intermediate-density lipoprotein as an independent risk factor for aortic atherosclerosis in hemodialysis patients. J Am Soc Nephrol 1998; 9:1277-1284.

13 Rostand SG, Kirk KA, Rutsky EA: Relationship of coronary risk factors to hemodialysisassociated ischemic heart disease. Kidney Int 1982;22:304-308.

14 Jungers P, Massy ZA, Khoa TN, Fumeron C, Labrunie M, Lacour B, Descamps Latscha B, Man NK: Incidence and risk factors of atherosclerotic cardiovascular accidents in predialysis chronic renal failure patients: A prospective study. Nephrol Dial Transplant 1997;12:2597_ 2602. 